\title{
PATIENT PARTENAIRE : DE LA PRATIQUE À LA RECHERCHE
}

\author{
Benoît Pétré, Gilles Louis, Bernard Voz, $\underline{\text { Alexandre Berkesse, Luigi Flora }}$
}

\author{
S.F.S.P. | « Santé Publique»
}

2020/4 Vol. 32 | pages 371 à 374

ISSN 0995-3914

Article disponible en ligne à l'adresse :

https://www.cairn.info/revue-sante-publique-2020-4-page-371.htm

Distribution électronique Cairn.info pour S.F.S.P..

(C) S.F.S.P.. Tous droits réservés pour tous pays.

La reproduction ou représentation de cet article, notamment par photocopie, n'est autorisée que dans les limites des conditions générales d'utilisation du site ou, le cas échéant, des conditions générales de la licence souscrite par votre établissement. Toute autre reproduction ou représentation, en tout ou partie, sous quelque forme et de quelque manière que ce soit, est interdite sauf accord préalable et écrit de l'éditeur, en dehors des cas prévus par la législation en vigueur en France. Il est précisé que son stockage dans une base de données est également interdit. 


\title{
Patient partenaire : de la pratique à la recherche
}

\section{Patient partner: from practice to research}

\author{
Benoît Pétré $^{1,2}$, Gilles Louis ${ }^{1,2}$, Bernard Voz ${ }^{1,2}$, Alexandre Berkesse ${ }^{3,4}$, Luigi Flora ${ }^{5,6}$
}

\section{$[\rightarrow$ Résumé}

L'approche du «patient partenaire de soins » est aujourd'hui considérée comme un levier majeur pour l'optimisation des systèmes de soins. Si les patients doivent indiscutablement être parties prenantes et critiques d'un système de santé, il n'en demeure pas moins que cette notion mérite d'être mieux documentée pour gagner en efficacité et efficience et asseoir de bonnes pratiques. Différents enjeux de recherche dans ce domaine sont soulevés sur des aspects de clarification conceptuelle, d'évaluation et de compréhension des pratiques. Cet article souhaite ouvrir le dialogue autour de la construction d'un agenda de recherche sur la thématique du partenariat.

Mots-clés: Participation du patient; Développement du système de santé ; Démocratie sanitaire ; Opinion d'expert.

\begin{abstract}
$\sqsubset$ Abstract
The "patient as care partner" approach is nowadays considered as a major lever for the optimization of health care systems. Although patients must undeniably represent major stakeholders of a healthcare system, this field of practice on partnership requires to be better documented in order to improve effectiveness and efficiency and to establish good practices. This article raises three main research issues in the field of partnership in health : conceptual clarification, evaluation and understanding of practices. This article aims to initiate a dialogue on the construction of a research agenda on the theme of partnership.
\end{abstract}

Keywords: Patient participation; Health system development; Health democracy; Expert opinion.

\footnotetext{
${ }^{1}$ Département des Sciences de la Santé publique - Université de Liège - Liège - Belgique.

${ }^{2}$ Consortium de recherche Approche patient-partenaire de soins - Programme Interreg VA Grande Région - Liège - Belgique.

${ }^{3}$ Pôle européen - Centre d'excellence sur le partenariat avec les patients et le public (CEPPP) - Montréal, Québec - Canada.

${ }^{4}$ Coordination pour l'amélioration des pratiques professionnelles en santé (Capps) Bretagne - Rennes - France.

${ }^{5}$ Centre d'innovation du partenariat avec les patients et le public (CI3P) - Université Côte d'Azur - Nice - France.

${ }^{6}$ École du partenariat - CEPP - Montréal, Québec - Canada.
} 


\section{Introduction}

L'organisation du système de santé contemporain rencontre des défis sans précédent - démographique (modification de la pyramide des âges), épidémiologique (progression des maladies chroniques), social (préoccupation des patients pour leurs droits et leur qualité de vie), technologique (accès en masse à l'information sanitaire) et économique (enveloppe budgétaire fermée) - appelant des modes de gouvernance nouveaux $[1,2]$. La reconnaissance de la participation du patient au premier plan de ses soins ainsi qu'avec les autres acteurs de la santé au sein des démarches d'amélioration du système de santé s'inscrit dans ces pratiques nouvelles d'approche de la santé [3]. Différentes perspectives coexistent aujourd'hui pour désigner cet engagement ou implication du patient. S'appuyant sur les travaux de Carman et al. [4], le modèle du « patient partenaire de soins » développé par l'université de Montréal - un des modèles les plus cités dans la littérature francophone - est relativement récent et propose une perspective d'intégration globale de l'engagement du patient dans le système de santé [5] : dispensation directe du ou des soin(s), organisation des services et de la gouvernance dans les établissements de santé, élaboration des politiques de santé, recherche et formation des professionnels de santé. Le modèle de Montréal propose un dépassement d'une approche dite "centrée sur le patient» en valorisant l'expertise issue du vécu de la maladie par le patient (explicitation des savoirs issus de leurs problèmes de santé ou psychosociaux et de leur utilisation du système de soins), en considérant le patient comme un membre à part entière - un partenaire - de l'équipe de soins $[5,6]$. Cette perspective vise à rééquilibrer les rapports au savoir, au contrôle et au pouvoir entre les professionnels de la santé et les malades ; les savoirs expérientiels des patients deviennent complémentaires et interdépendants des savoirs scientifiques des professionnels de santé [7].

L'intérêt des acteurs du monde de la santé à l'égard de cette thématique semble grandissant et tend à se renforcer au vu de l'accumulation progressive, mais encore limitée, des preuves d'efficacité de l'approche de partenariat sur l'efficacité et l'efficience des soins [8]. Cet article profite d'une communication réalisée lors du ${ }^{\mathrm{er}}$ Colloque international sur le partenariat de soins avec les patients en France [9] et se donne comme objectif de suggérer quelques pistes de recherche sur la thématique du partenariat de soins et d'ouvrir un appel à la constitution d'un agenda de recherche partagé.

\section{Quelles recherches sur le partenariat ?}

Le partenariat des soins constitue un thème de recherche de plus en plus prégnant. Au cours des dernières années, plusieurs auteurs ont ainsi tenté d'analyser le flux de publications autour de cette thématique dont il est possible de tirer quelques constats $[10,11]$. Il ressort en premier lieu de ces travaux que la recherche dans ce domaine s'est intensifiée au cours des vingt dernières années. À titre d'exemple, Barrelo et al. [10] font état d'une multiplication par douze du nombre d'articles publiés entre 2002 et 2011 relatifs à l'engagement des patients. La majorité des articles sont publiés par des équipes nord-américaines suivies des européennes, principalement par des acteurs issus de la médecine et des sciences infirmières. Les approches interdisciplinaires restent marginales en Europe. Les travaux sur l'engagement des patients restent également disproportionnés comparativement à ceux centrés sur l'adhésion thérapeutique des patients, montrant la finalité qui anime, de manière toujours prédominante, le domaine de la santé.

Nous appelons des travaux de recherche sur trois axes qui nous paraissent particulièrement utiles pour avancer des pratiques aux bonnes pratiques et documenter/ soutenir les propositions d'intervention dans le domaine : la clarification des concepts mobilisés, l'évaluation et la compréhension des pratiques.

\section{Une nécessaire clarification conceptuelle}

L'observation la plus marquante est certainement le caractère polysémique du partenariat. On relève une terminologie hétérogène pour désigner cette participation du patient aux soins : «empowerment», "engagement», «involvement», «partenariat» sont autant de vocables retrouvés dans la littérature pour désigner une forme de contribution du patient à ses soins ou au service du système de santé. Si tous ces termes renvoient à une intention de permettre aux patients d'être acteurs de leurs soins ou du système de santé, ils sont toutefois rarement combinés dans un même travail de recherche montrant que les termes ont une signification particulière et renvoient à un champ d'actions spécifiques $[10,11]$. Sans nier les enjeux liés à l'épistémologie et aux origines mêmes des différents concepts utilisés, le peu de consensus, le recouvrement partiel entre les notions et les frontières étroites, voire poreuses entre les concepts entraînent une confusion potentielle auprès des acteurs de terrain et la mise en 
pratique. Des travaux de clarification existent, notamment ceux d'Halabi et al. [12] qui proposent une lecture intégrative des concepts et déclinent de manière cumulative les composantes du partenariat de soins. Les assises sur lesquelles repose le modèle de patient partenaire demandent toutefois à être consolidées. Par exemple, Barello et al. [13] appellent à documenter le processus d'engagement, arguant que cet engagement du patient dans les soins qu'il reçoit ne peut être considéré comme binaire (être patient-partenaire à un temps $t$, et ne plus l'être à $t+1$ ) et devrait considérée en relation avec le développement psychologique du sujet à l'endroit de sa maladie. Gross et Gagnayre [14] proposent quant à eux une taxonomie de savoirs apportés par les patients au service de la formation des professionnels de santé venant affiner la description du savoir expérientiel développé par Flora [15] et repris dans le modèle de Montréal.

En Europe francophone, le partenariat devra particulièrement être discuté au regard des approches en matière d'éducation thérapeutique du patient (ETP), la plupart de ces dispositifs étant plus fréquemment caractérisés par une approche centrée sur le patient que par une approche de partenariat avec les patients. En effet, Voyen et al. [9] indiquent que le concept de «partenariat» est mal connu des acteurs de l'ETP et que l'ETP est considérée comme un dispositif pédagogique et opérationnel au service du partenariat.

\section{Une évaluation des pratiques}

Un deuxième axe de recherche est celui de l'évaluation des interventions se déclarant comme relevant du partenariat. Dès leur communication sur le modèle de Montréal, les concepteurs ont plaidé pour une évaluation systématique des démarches de partenariat [6]. Force est de constater la rareté des évaluations formelles de ces démarches [16], amenant à considérer le partenariat avec un niveau de preuve scientifique ou académique dit « faible».

D'autres chercheurs appellent également à dépasser l'évaluation des effets et de l'impact du partenariat vers des questions portant sur le processus de développement de ce partenariat [13]. Il ne s'agit pas seulement de fournir une preuve d'efficacité, mais également de réfléchir aux modèles, aux formations et aux interventions les plus fécondes.

Plusieurs propositions peuvent être formulées aujourd'hui pour mieux comprendre et consolider les pratiques: une compilation de l'existant sous forme de synthèse de type «méta-analyse » ou « revue narrative » recoupant les différentes disciplines est attendue; de nouveaux modèles d'évaluation dépassant les propositions des disciplines biomédicales sont appelés. Les démarches de patient partenaire peuvent être caractérisées d'«intervention complexe » et demandent d'autres standards d'évaluation que les fameux «essais contrôlés randomisés». Des inspirations dans des méthodes d'évaluation dites « réalistes", largement mobilisées en sciences humaines et sociales doivent permettre de mieux situer quels contextes, quels mécanismes permettent d'obtenir certains résultats [17]. Il s'agit également de mieux définir les critères et indicateurs d'évaluation dans une perspective écosystémique/intégrative du patient partenaire. Les effets sur les patients, sur les professionnels de santé, sur le système de santé lui-même doivent être pris en considération, sans oublier les limites et effets pervers possibles de ces interventions.

\section{Compréhension des pratiques}

Le troisième axe que nous avons souhaité exposer est lié au changement de culture que requiert et qu'induit le patient partenaire. D'un côté, le partenariat vient bousculer les rapports sociaux et les institutions, amenant au besoin de mieux comprendre ce qui est en jeu dans ces pratiques innovantes : comment les relations de pouvoir et d'identité que l'on dit modifier sont-elles effectivement modifiées? Quelle identité pour les nouvelles figures professionnelles "patients »? Patients «formateurs», « chercheurs», etc. suscitent de nombreuses questions et débats : quelle place leur donner? Faut-il professionnaliser leur recrutement, leur formation et leurs contributions? De l'autre côté, on peut également se demander comment les professionnels de santé évoluent et conservent une autonomie et légitimité professionnelle, confrontés à des situations de travail ouvertes aux patients.

De manière plus globale, il convient de mieux situer comment notre système de santé et son organisation sont prêts à accueillir et développer ces pratiques de partenariat. C'est ce que tente de réaliser un consortium de recherche composé d'équipes belges, françaises, allemandes et luxembourgeoises dans le cadre du projet «Approche patient partenaire de soins » (APPS) [18] financé par le Fonds européen de développement régional (Feder) dans le territoire de la Grande Région (GR). S'appuyant sur une logique d'échanges interrégionaux, les acteurs du projet cherchent à identifier quelles sont les conditions de déploiement durable du partenariat, répondant aux caractéristiques légales, culturelles, sociales 
et économiques de ladite GR. C'est ici un axe de recherche sur l'implémentation du partenariat qui est ouvert.

\section{Ouverture vers le développement d'un agenda de recherche}

Le modèle de partenariat de soins gagne en notoriété partout dans le monde et particulièrement en Europe qui y voit un moyen d'évolution durable du système de santé. S'il est indiscutable que les bénéficiaires d'un système de santé et de soins doivent participer à la construction de ce même système, il convient aujourd'hui de définir les meilleures conditions de partenariat. La recherche doit être renforcée pour consolider et enrichir les connaissances sur la thématique et lui faire valoir ses lettres de noblesse. Il s'agit de passer des pratiques, aux bonnes pratiques.

Ce texte est loin de couvrir l'exhaustivité des besoins de recherche appelés sur la thématique du partenariat de soins. Il doit avant tout être considéré comme un appel à la communauté scientifique en vue de l'élaboration d'un agenda de recherche. Il convient de poursuivre le développement de cet agenda, qui devrait lui-même suivre les principes du travail en partenariat, notamment à travers la coconstruction avec des patients et un ancrage interdisciplinaire.

Le projet APPS est cofinancé par le Fonds européen de développement régional dans le cadre du programme INTERREG VA Grande Région. Il est soutenu par les autorités publiques des quatre régions (Wallonie, Grand-Duché du Luxembourg, Lorraine, Rhénanie-Palatinat et Sarre).

\section{Références}

1. Anderson RM, Funnell MM. Patient empowerment: Myths and misconceptions. Patient Educ Couns. 2010;79(3):277-82.

2. Maclntosh E, Rajakulendran N, Khayat Z, Wise A. Transforming health: Shifting from reactive to proactive and predictive care [Internet]. 2016 Mars 29. [Visité le 11/11/2017]. En ligne: https://bit.ly/2ldlvwF.
3. Richards T, Montori VM, Godlee F, Lapsley P, Paul D. Let the patient revolution begin. BMJ. 2013;346:f2614.

4. Carman KL, Dardess P, Maurer M, Sofaer S, Adams K, Bechtel C, et al. Patient and family engagement: $a$ framework for understanding the elements and developing interventions and policies. Health Aff (Millwood). 2013;32(2):223-31.

5. Pomey MP, Flora L, Karazivan P, Dumez V, Lebel P, Vanier MC, et al. Le "Montreal model ": enjeux du partenariat relationnel entre patients et professionnels de la santé. Santé Publique. 2015;S1(HS):41-5.

6. Karazivan P, Dumez V, Flora L, Pomey MP, Del Grande C, Guadiri S, et al. The patient as-partner approach in health care: a conceptual framework for a necessary transition. Acad Med. 2015;90(4): 437-41.

7. Flora L, Berkesse A, Payot A, Dumez V, Karazivan P. L'application d'un modèle intégré de partenariat dans la formation des professionnels de la santé : vers un nouveau paradigme humaniste et éthique de coconstruction des savoirs en santé. J Int Bioéthique. 2016;27(1):59-72.

8. Boivin A, Lehoux P, Lacombe R, Burgers J, Grol R. Involving patients in setting priorities for healthcare improvement: a cluster randomized trial. Implementation Sci. 2014;9:24.

9. Voyen M, Böhme P, Germain L, Pétré B, Younsi M, Mathieu J, et al. Connaissance, perception et définition du concept " patient partenaire de soins ": Qu'en pensent les acteurs impliqués en éducation thérapeutique? Résultats d'une enquête nationale. Ier Colloque international sur le partenariat de soin avec le patient en France. Nice, 14-15 oct. 2019. Le partenariat de soin avec le patient: Analyses. 2020;1:73-86. [Visité le 23/08/2020]. En ligne: https://partenariat.sciencesconf.org/.

10. Barello S, Graffigna G, Vegni E. Patient engagement as an emerging challenge for healthcare services: mapping the literature. Nurs Res Pract. 2012;2012:905934.

11. Menichetti J, Libreri C, Lozza E, Graffigna G. Giving patients a starring role in their own care: a bibliometric analysis of the on-going literature debate. Health Expect. 2014;19(3):516-26.

12. Halabi IO, Scholtes B, Voz B, Gillain N, Durieux N, Odero A, et al. "Patient participation" and related concepts: A scoping review on their dimensional composition. Patient Educ Couns. 2020;103(1):5-14.

13. Barello S, Graffigna G, Savarese M, Bosio AC. Engaging patients in health management: towards a preliminary theoretical conceptualization. Psicologia Della Salute. 2014;3:11-33.

14. Gross O, Gagnayre R. Caractéristiques des savoirs des patients et liens avec leurs pouvoirs d'action : implication pour la formation médicale. Revue française de pédagogie. 2017;201(4):71-82.

15. Flora L. Savoirs expérientiels des malades, pratiques collaboratives avec les professionnels de santé : état des lieux. Éducation permanente. 2013;195(2):59-72.

16. Rahimi S, Zomahoun HTV, Légaré F. Patient engagement and its evaluation tools: Current challenges and future directions. Int J Health Policy Manag. 2019;8(6):378-80.

17. Pawson R, Tilley N. Realistic evaluation. London: Sage; 1997. 36 p.

18. Pétré B, Scholtes B, Voz B, Ortiz I, Gillain N, Husson E, et al. L'approche patient partenaire de soins en question. Rev Med Liege. 2018;73(12):620-5. 